\title{
Dorsomedial prefrontal cortex 5-HT6 receptors regulate anxiety-like behavior
}

\author{
Fei Geng ${ }^{1} \cdot$ Jia Tian $^{2} \cdot$ Jian-Lin Wu ${ }^{3} \cdot$ Yun Luo ${ }^{1} \cdot$ Wen-Jun Zou ${ }^{3} \cdot$ Chao Peng ${ }^{3} \cdot$ Gui-Feng Lu $^{4}$
}

Published online: 4 December 2017

(C) Psychonomic Society, Inc. 2017

\begin{abstract}
The dorsomedial prefrontal cortex (dmPFC) plays a very important role in decision-related and anxiety-related information processing. It has enriched 5-HT6 receptors; however, the precise role of dmPFC 5-HT6 receptors in anxiety remains to be fully investigated. In this study, we injected dmPFC with the 5-HT6 receptor agonist EMD 386088 and antagonist SB 271046 using stereotactic technology. 5HT6 receptor activation in mice increased time spent in the center area on the open-field test, increased exploration of the open arms on the elevated plus maze test, and increased ratio on the social interaction test. 5-HT6 receptor inactivation induced the opposite effects. In brain slices, EMD 386088 decreased both spontaneous inhibitory postsynaptic currents (sIPSC) and spontaneous excitatory postsynaptic currents (sEPSC), while SB 271046 only increased sEPSC. These effects of EMD 386088 and SB 271046 could be reversed by the $\mathrm{GABA}_{\mathrm{A}}$ receptor antagonist bicuculline (BMI) and positive allosteric modulator clonazepam (CLZ), respectively. Our results suggest that neurotransmission in the dmPFC by 5-HT6 receptor activation and inhibition may play an important role in anxiety-like behavior, and may provide new insight into the pathological mechanism and potential target of anxiety disorders.
\end{abstract}

Keywords dmPFC $\cdot$ EMD $386088 \cdot \mathrm{SB} 271046 \cdot 5$-HT6 receptor $\cdot$ Anxiety

The medial prefrontal cortex (mPFC) is the region of the human brain that can be activated by emotional stimuli (Kober et al., 2008); activation or depression of the $\mathrm{MPFC}$ has a profound influence on emotional responses (Vogt, Finch, \& Olson, 1992). The mPFC consists two important regions: the ventral portion (vmPFC) and the dorsal portion, which includes the dorsal anterior cingulate cortex (dmPFC) (Haber \& Behrens, 2014; Rushworth \& Behrens, 2008). The $\mathrm{dmPFC}$ is important for decision-making and expression of fear or anxiety (Courtin et al., 2014; Maier et al., 2012; Rushworth, Noonan, Boorman, Walton, \& Behrens, 2011). For example, in interoceptive perception, recruitment of the

Gui-Feng Lu

emptylgf@126.com

1 Department of Physiology, Zunyi Medical College, Zunyi 563003, China

2 College of Biological Science and Technology, Heilongjiang BaYi Agriculture University, Daqing 163319, China

3 Department of Neurobiology, Southern Medical University, Guangzhou 510515, China

4 Department of Pathophysiology, Zunyi Medical College, Zunyi 563003, China
dmPFC is correlated with anxiety levels (Haber \& Behrens, 2014).

The 5-HT6 receptor is a type of 5-HT receptor superfamily coupled with a Gs-sensitive AC5 isoform, which leads to 3'5'cyclic adenosine monophosphate (cAMP) production (Ruat et al., 1993). Previous studies showed its highest expression in the striatum, cortex, and nucleus accumbens of an adult animal's brain (Brailov et al., 2000; Hamon et al., 1999). In several brain regions, the 5-HT6 receptor is localized at the postsynaptic neuron (Tassone et al., 2011). The functional roles of 5-HT6 receptors have been studied over the past 15 years. Some studies focused on the physiological role this receptor has on learning and memory (Mitchell \& Neumaier, 2005; Rogers \& Hagan, 2001), while others have shown evidence that 5-HT6 receptors are involved in feeding behavior (Heal, Smith, Fisas, Codony, \& Buschmann, 2008). Additionally, 5-HT6 receptor antagonists were developed as a treatment for cognitive impairment associated with schizophrenia and obesity (Pogacic Kramp, 2012).

A large number of studies have focused on anxiety in the past few years. At first glance, paradoxically, 5-HT6 receptor agonists induced similar effects to 5-HT6 receptor antagonists in animal models of anxiety (Carr, Schechter, \& Lucki, 2011; Nikiforuk, Kos, \& Wesolowska, 2011; Schechter et al., 2008; 
Wesolowska \& Nikiforuk, 2007); however, the anxiolytic responses induced by the 5-HT6 receptor agonist EMD 386088 could be blocked by antagonist SB 271046 (Nikiforuk et al., 2011). It is possible that 5-HT6 receptor agonists and antagonists happen to produce similar behavioral effects through different neurochemical mechanisms or actions mediated in different brain regions. Nikiforuk et al. (2011) demonstrated the role of the rat hippocampus 5-HT6 receptor on anxiety behaviors.

The aim of the present study was to investigate the local roles of 5-HT6 receptors in animal models of anxiety. We injected the 5-HT6 receptor agonist EMD 386088 and antagonist SB 271046 into the dorsomedial prefrontal cortex (dmPFC) using the stereotactic technology. 5-HT6 receptor activation induced anxiolytic effects, while 5-HT6 receptor inactivation induced opposite effects. These effects could be reversed by the $\mathrm{GABA}_{\mathrm{A}}$ receptor antagonist bicuculline (BMI) and positive allosteric modulator clonazepam. Our results suggest that imbalances of neurotransmission in the dmPFC by 5 -HT6 receptor activation and inhibition may play an important role in anxiety-like behavior and may provide new insight into the treatment of anxiety disorders.

\section{Method}

\section{Animals}

At the start of the experiment, male C57 BL/6J mice (8-9 weeks, four or five per cage) were housed with a 12-hr light/ dark cycle (lights on at 8:00 a.m.) and given food and water ad libitum under standard conditions $\left(21-26^{\circ} \mathrm{C}\right)$. Behavioral testing was performed from 12:00 to 17:00, and all aspects of the behavioral experiment followed the rules of the Chinese Council on Animal Care. Animal suffering was minimized through our efforts.

\section{Reagents}

The following drugs were used: D(-)-2-Amino-5phosphonopentanoic-acid (AP5, 100 $\mathrm{M}$; Sigma-Aldrich, USA) and 6-cyano-7-nitroquinoxaline-2,3-dione (CNQX, $20 \mu \mathrm{M}$; Tocris Bioscience, UK). The 5-Chloro-2-methyl3-(1,2,3,6-tetrahydro-4-pyridinyl)-1H-indole hydrochloride (EMD 386088 or EMD) and 5-chloro-N-[4-methoxy-3-(1piperazinyl)phenyl]-3-

methyl-benzothiophene-2-sulfonamide (SB 271046 or SB) were synthesized by Adamed (Pienkow, Poland). EMD and SB were dissolved in distilled water. AP5 and CNQX were dissolved in dimethyl sulfoxide (DMSO), and the concentration of DMSO (dissolved in water, Sigma) was $<0.1 \%$. Bicuculline methiodide (BMI; TocrisBioscience, UK) was dissolved in water. All other chemicals were from Sigma.
Clonazepam $(0.0625 \mathrm{mg} / \mathrm{kg} \sim 0.5 \mathrm{mg} / \mathrm{kg}$, Sigma) was diluted in PBS (with $0.5 \%$ methylcellulose), and the drug administered by intraperitoneal injection. The injection volume $0.2 \mathrm{ml} / 10 \mathrm{~g}$ was made $30 \mathrm{~min}$ before behavioral testing. Weights and concentration used for 5-HT6 agonist/ antagonist and Bicuculline were $2.5 \mu \mathrm{g}, 03-2.4 \mu \mathrm{g}$, and $0.2 \mathrm{mM}$ (i.dmPFC), respectively.

\section{Intracerebral infusions}

We used pentobarbital sodium $(50 \mathrm{mg} / \mathrm{kg}$ ) to anesthetize the mice. The mice were then placed on stereotaxic instruments (Stoelting, IL, USA), and the related surgeries were performed to expose a small burr hole (1-mm diameter) in the skull of the mice. In accordance with the following coordinates (dmPFC): anterior $1.9 \mathrm{~mm}$; lateral $0.4 \mathrm{~mm}$; ventral $1.5 \mathrm{~mm}$ (Courtin et al., 2014), stainless-steel cannulas (Plastics One, INC; C315G/SPC; length, $1.8 \mathrm{~mm}$ ) were randomly lowered (unilaterally) into the dmPFC. Glass ionomer cements were used to fix the cannulas, and the mice were allowed to recover on an electric blanket. After surgery, the mice were sent back into home cages for recovery for the following 7 days.

Before the behavioral tests, intra-dmPFC injections were performed through infusion cannulas (Plastics One, INC; C315I/SPC, length matched to the guide cannulas). The infusion cannulas were connected to $10-\mu 1$ microsyringes (Hamilton, Reno, NV, USA) via polyethylene tubing (Plastics One, INC; C313C). Intra-dmPFC infusion $(0.5 \mu \mathrm{l} /$ site) was performed at a rate of $0.2 \mu \mathrm{l} / \mathrm{min}$. The mice and infusion cannula were held in position for 3 minutes to allow diffusion. Mice were randomly assigned to groups ( $n=10$ or 11 /group) according to intra-dmPFC treatment. The interval between behavioral tests was 2 days. The interval between drug infusion and behavioral testing was 20 minutes. The different drugs administered in combination were given together at the same time, and the control groups were injected with artificial cerebrospinal fluid.

\section{Open-field test}

Briefly, mice were habituated in the testing house for $1 \mathrm{~h}$ before behavioral testing, then each individual mouse was placed in the center of chamber $(40 \times 40 \times 30 \mathrm{~cm})$. Twentyfive-W halogen bulbs were used to illuminate the open field area. During a 5-minute recording period, mice were gently placed and monitored with a Versamax animal behavior monitor (AccuScan, Columbus, OH, USA). Time that mice spent in the center $(15 \times 15 \mathrm{~cm}$ square $)$ and the total distance that mice explored in the open field were measured, and $20 \%$ ethanol was used to clean the open field arena between each trial. 


\section{Elevated plus maze test}

The plus-shaped elevated plus maze was raised $50 \mathrm{~cm}$ above the floor. The apparatus consisted of two closed arms $(25 \times 8 \times$ $0.5 \mathrm{~cm})$ and two open arms $(25 \times 8 \times 12 \mathrm{~cm})$ that extended from a central platform $(8 \times 8 \mathrm{~cm})$. Closed arms were surrounded by $12-\mathrm{cm}$ high transparent walls and open arms were surrounded by a $0.5 \mathrm{~cm}$ ledge to prevent falling from the maze. Each mouse was placed in the center facing a closed arm. A camera was used to record the movement of the mouse during a 5-minute period. Total distance and time spent in open arms and closed arms were measured. The maze was cleaned with $20 \%$ ethanol between each trial.

\section{Social interaction test}

The social interaction chamber consists of a large open field $(89 \times 63 \times 60 \mathrm{~cm})$ containing a small wire cage $(14 \times 17 \times$ $14.5 \mathrm{~cm})$. Mice were habituated to the testing house. An unfamiliar male mouse was introduced into the wire cage. We recorded the amount of time the test mouse spent interacting with the wire cage during the two 2.5 minute phases (with and without the unfamiliar C57 BL/6J mouse). The ratio between these two phases was defined as the interaction index (Geng et al., 2016).

\section{Brain-slice electrophysiology}

Slices were prepared from the mice aged 7-8 weeks. Mice were decapitated after being anesthetized with ether, and the brain was quickly removed in ice-cold artificial cerebrospinal fluid (ACSF, cutting solution) made of $220 \mathrm{mM}$ sucrose, $2.5 \mathrm{mM} \mathrm{KCl}, 1.3 \mathrm{mM} \mathrm{CaCl}_{2}, 2.5 \mathrm{mM} \mathrm{MgSO}_{4}, 1 \mathrm{mM}$ $\mathrm{NaH}_{2} \mathrm{PO}_{4}, 26 \mathrm{mM} \mathrm{NaHCO}$, and $10 \mathrm{mM}$ glucose. Horizontal slices $(300 \mu \mathrm{m})$ were cut with a vibrating microtome (Leica VT 1000S, Germany). Slices were then transferred into a chamber for recovery in $32^{\circ} \mathrm{C}$. The chamber contained the recording ACSF, made of $126 \mathrm{mM} \mathrm{NaCl}$, $26 \mathrm{mM} \mathrm{NaHCO} 3,3 \mathrm{mM} \mathrm{KCl}, 1.2 \mathrm{mM} \mathrm{NaH}_{2} \mathrm{PO}_{4}, 2 \mathrm{mM}$ $\mathrm{CaCl}_{2}, 1 \mathrm{mM} \mathrm{MgSO}_{4}$, and $10 \mathrm{mM}$ glucose. All solutions were bubbled with $95 \% \mathrm{O}_{2} / 5 \% \mathrm{CO}_{2}$ ( $\mathrm{vol} / \mathrm{vol}$ ).

Slices were moved to a recording chamber, and the recording solution (ACSF) was perfused through the chamber at a flow rate of $3 \mathrm{ml} / \mathrm{min}$. Spontaneous IPSCs (sIPSCs) were recorded at a holding potential of $-70 \mathrm{mV}$, with an internal solution containing $140 \mathrm{mM} \mathrm{CsCl}, 2 \mathrm{mM} \mathrm{MgCl} 2,1 \mathrm{mM}$ $\mathrm{CaCl}_{2}, 10 \mathrm{mM}$ EGTA, $10 \mathrm{mM}$ HEPES-CsOH, $2 \mathrm{mM}$ adenosine triphosphate, and $5 \mathrm{mM}$ QX-314. One mM kynurenic acid was added to the recording solution. Spontaneous EPSCs (sEPSCs) were recorded after application of the BMI $(20 \mu \mathrm{M})$. To record sEPSCs, the pipettes contained $105 \mathrm{mM}$ Kgluconate, $30 \mathrm{mM} \mathrm{KCl}, 10 \mathrm{mM}$ HEPES, $10 \mathrm{mM}$ phosphocreatine, 4 mM ATP-Mg, 0.3 mM GTP-Na, 0.3 mM EGTA, and $5 \mathrm{mM}$ QX314 (pH 7.4, 290 mOsm). BMI, CNQX and AP5 were used to block inhibitory and excitatory currents, respectively. EMD $(20 \mu \mathrm{M})$ and SB $(10 \mu \mathrm{M})$ were used in vitro brain slices. Glass pipettes, Digidata1440 converter and Axoclamp-700B amplifiers (Molecular Devices, Sunnyvale, CA) were used. All reagents were purchased from Sigma.

\section{Statistical analysis}

All data were shown as the mean \pm standard error of the mean (SEM) and analyzed using the Student's $t$ test and one-way ANOVA, with a least squares difference (LSD) test for post hoc comparisons. A two-way ANOVA was performed to analysis the interaction of two drugs. All the statistical analyses were done using SPSS software (SPSS, Inc.), and the value ( $p$ $<.05)$ was considered to represent statistical significance.

\section{Results}

\section{Activation of the 5-HT6 receptor in the dmPFC induces anxiolytic effects}

Figure 1a demonstrates that the cannulas were implanted into the dmPFC, and trypan blue staining showed that the spread of drugs was limited into the dmPFC. In the openfield test, mice injected with EMD (Nikiforuk et al., 2011) spent more time in the center of the open field $(t=-2.7, p$ $=.0146$; see Fig. 1b), although the total distance covered by EMD mice showed no significant difference compared with the control mice ( $t=0.842, p=0.411$; see Fig. $1 \mathrm{c})$, which excluded differences in motor ability. During a 5min elevated plus maze test, EMD mice spent more time in the open arms $(t=-2.85, p=.0107$; see Fig. 1d), while the velocity had no significant difference compared with the control mice $(t=0.781, p=.445$; see Fig. 1e). The control mice were defined as mice who received the ACSF infusion into the dmPFC. In addition, EMD mice showed profound desire for interaction in the social interaction test ( $t=-3.89, p=.00109$; see Fig. 1f). These results demonstrate that activation of dmPFC 5-HT6 receptors showed anxiolytic effects in mice.

\section{Inhibition of 5-HT6 receptors in the dmPFC induces anxiety-like behavior}

In order to confirm the active dose of 5-HT6 receptor antagonist $\mathrm{SB}$, dose-response analyses of SB were performed. When the concentration of SB was more than $0.6 \mu \mathrm{g}$, mice decreased their time in the central area of the open field compared to the control mice, $F(2,29)=4.97, p=.015$ (see Fig. 2a). SB infusion into the dmPFC did not affect their overall activity 
a

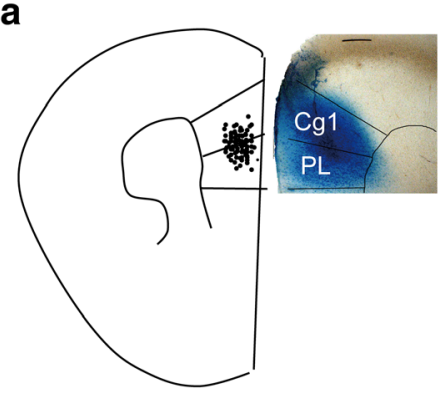

d

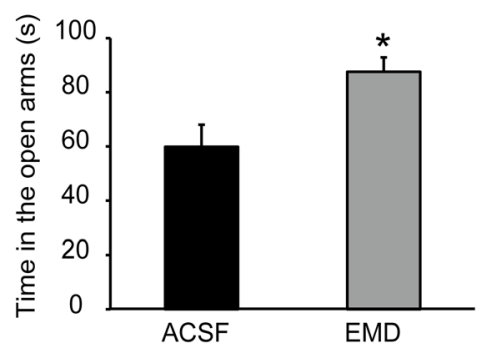

b

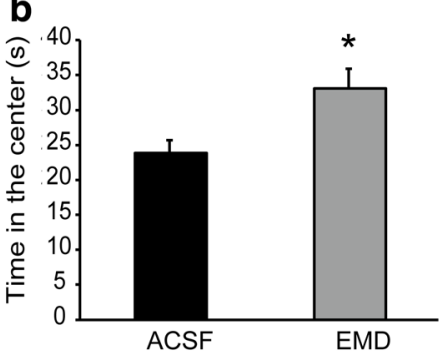

e

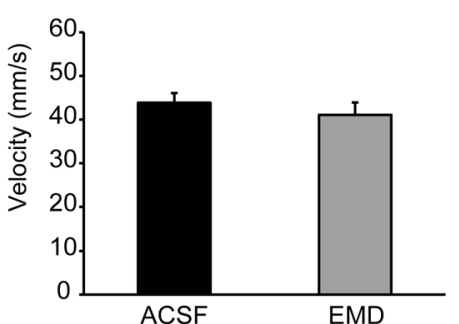

C

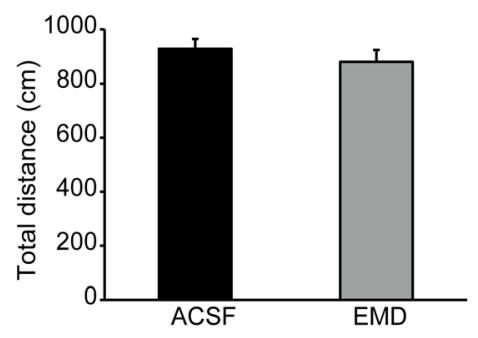

f

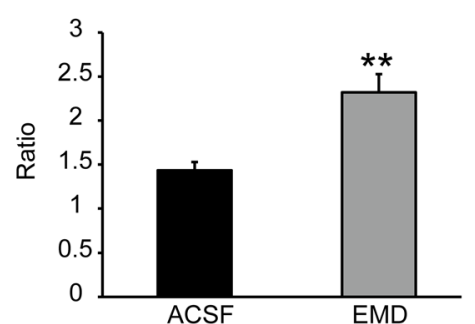

Fig. 1 Activation of 5-HT6 receptors in the dmPFC induces anxiolytic effects. a Left: infusion cites $(n=88)$. Right: representative infusion site by the trypan blue staining $(300 \mu \mathrm{m})$. b-d Mice tested 20 min after EMD infusion spent more time in the center area of the open field test (b) and more time exploring the open arms of the elevated plus maze (d) than

controls; however, no difference in total traveling distance was observed (c). e The velocity that mice travelled at in the elevated plus maze. f The ratio in the social interaction test was increased in the EMD-treated group. Data are presented as the mean $\pm S E . n=10$ mice per group. $* p<.05, * * p$ $<.01$ versus ACSF, Student's $t$ test

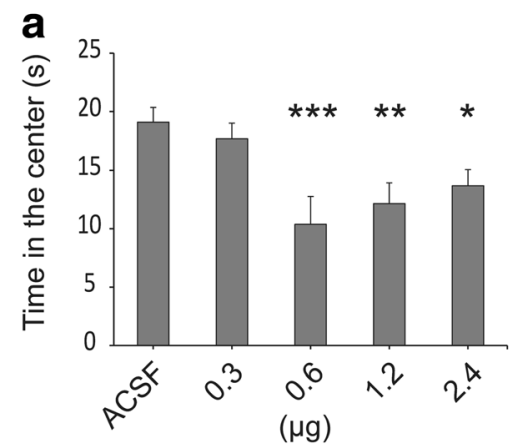

b
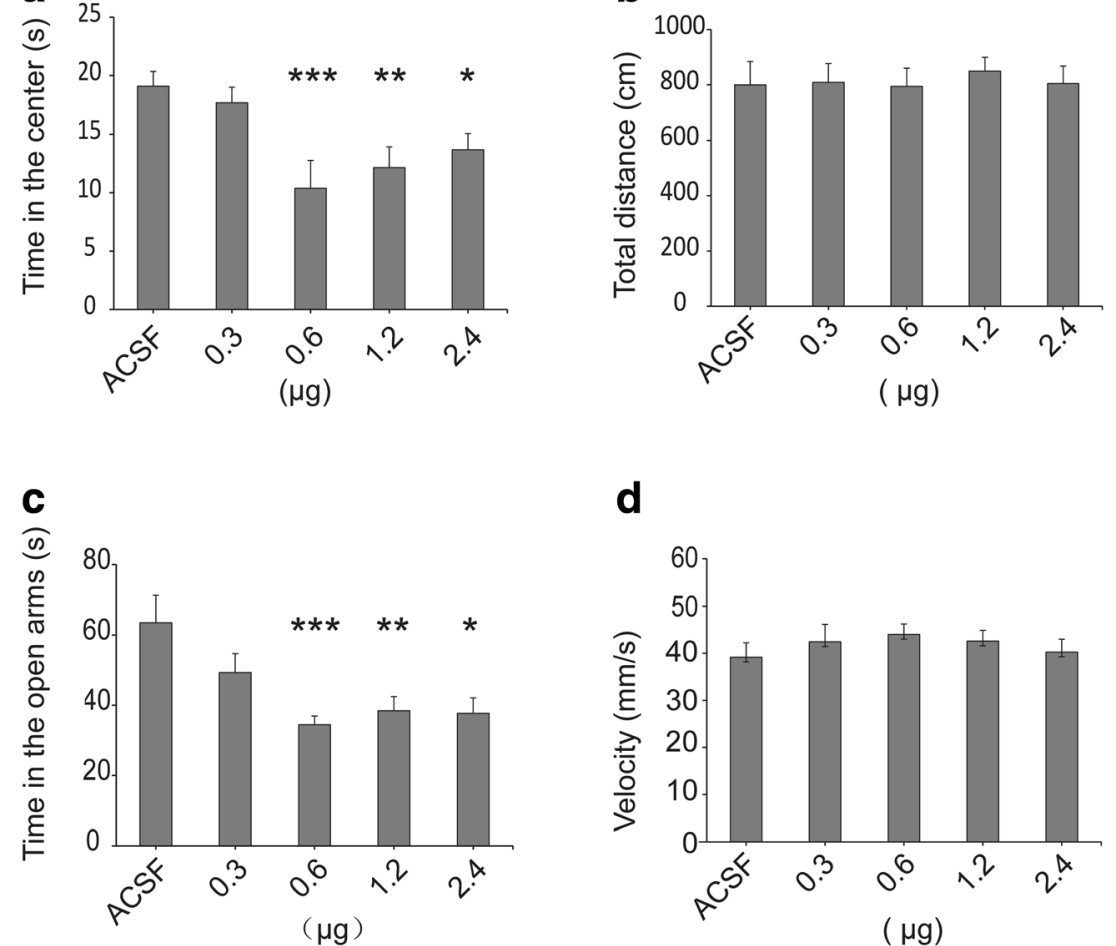

Fig. 2 Depression of 5-HT6 receptors in the dmPFC induces anxiety-like behavior. a Time spent in the center in the open field test. $\mathbf{b}$ Total distance. c Time spent in the open arms of the elevated plus maze. $\mathbf{d}$ The velocity

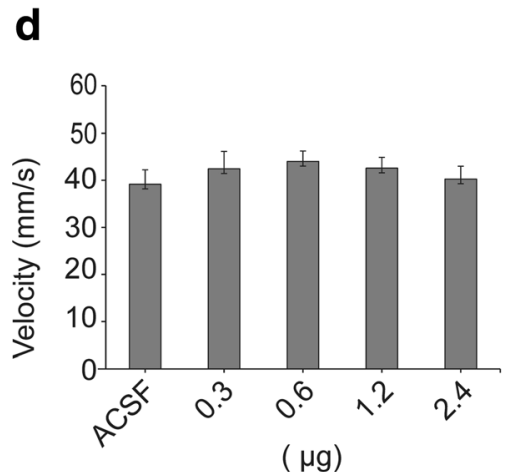

that mice travelled at in the elevated plus maze. Data are presented as the mean $\pm S E . n=10$ or 11 mice per group. $* p<.05, * * p<.01$ versus ACSF, one-way ANOVA/LSD post hoc test 
in the open-field test, $F(2,29)=0.019, p=.981$ (see Fig. 2b). Furthermore, mice that were subjected to the elevated plus maze after SB infusion spent less time in the open arms compared to control mice, $F(2,29)=4.37, p=.023$ (see Fig. 2 c), while the velocity was not affected, $F(2,29)=1.46, p=.25$ (see Fig. 2d). Dose of SB within 0.6 and $2.4 \mu \mathrm{g}$ was selected to conduct the following experiments. The results showed that blockade of 5-HT6 receptors induced anxiogenic-like activity.

\section{Activation of 5-HT6 receptors increased relative GABAergic neurotransmission in cortical pyramidal neurons}

Some forms of anxiety are postulated to be caused by an imbalance of synaptic transmission between excitatory and inhibitory circuits. In the presence of EMD, the frequency of spontaneous EPSCs in dorsomedial prefrontal cortex slices was unchanged, but the amplitude of spontaneous EPSCs decreased, $F(2,23)=0.186, p=.832$ (see Fig. 3a); $F(2,23)=$ $7.56, p=.0009$ (see Fig. 3b). Furthermore, the frequency of spontaneous IPSCs in dorsomedial prefrontal cortex slices was unchanged, but the amplitude of spontaneous IPSCs decreased, $F(2,23)=0.401, p=.674$ (see Fig. 3c); $F(2,23)=$ 4.801, $p=.005$ (see Fig. 3d). The above results show that EMD decreased the levels of EPSCs and IPSCs, thus indicating that the involvement of GABAergic neurotransmission

a
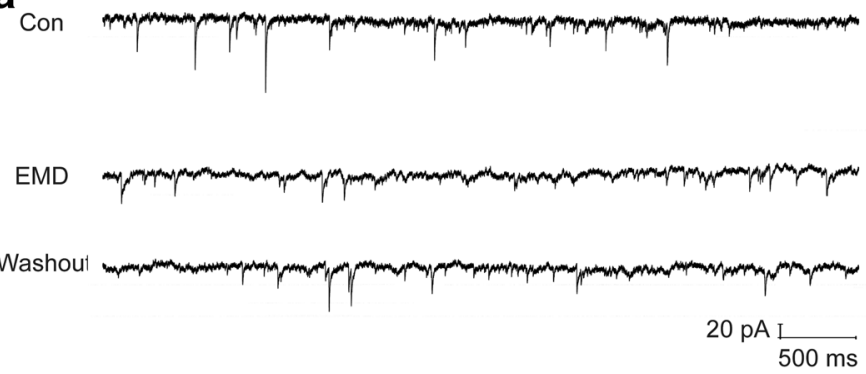

C

Con

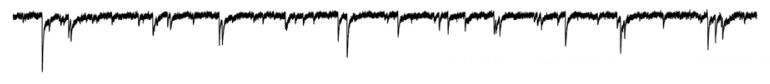

EMD

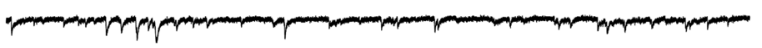

Washout

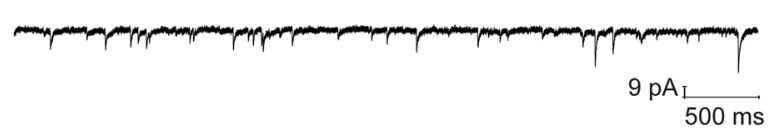
and glutamate transmission.

Inactivation of 5-HT6 receptors increased the excitatory inputs in cortical pyramidal neurons

We tested the effects of SB on GABAergic inhibitory transmission in the dmPFC pyramidal neuron. In the presence of $\mathrm{SB}$, the frequency of spontaneous EPSCs in dorsomedial prefrontal cortex slices was unchanged, but the amplitude of spontaneous EPSCs increased, $F(2,11)=0.963, p=.404$ (see Fig. 4a); $F(2$, $11)=4.16, p=.016$ (see Fig. 4b). Meanwhile, the frequency of spontaneous IPSCs in dorsomedial prefrontal cortex slices was unchanged, and the amplitude of spontaneous IPSCs was also unchanged, $F(2,13)=0.0729, p=.93$ (see Fig. $4 \mathrm{c}$ ); $F(2,13)=$ $0.0484, p=.953$ (see Fig. 4d). These results support our hypothesis that relative excitatory synaptic activity increases as a consequence of 5-HT6 receptor inhibition.

\section{Rescue of anxiolytic behavior induced by EMD via BMI infusion}

Intra-mPFC administration of the $\mathrm{GABA}_{\mathrm{A}}$ receptor antagonist BMI induced significant anxiogenic-like behavior (Solati, Hajikhani, \& Golub, 2013). To investigate how 5-HT6 receptors participate in anxiety and its role with $\mathrm{GABA}_{\mathrm{A}}$ receptors, mice were randomly allocated into four groups based on treatment (ACSF, EMD, EMD + BMI, BMI). We compared all combinations in a single ANOVA, with treatment drug as the fixed factor, which showed a significant effect. Paired comparisons were then performed. Disinhibition of dmPFC by BMI b
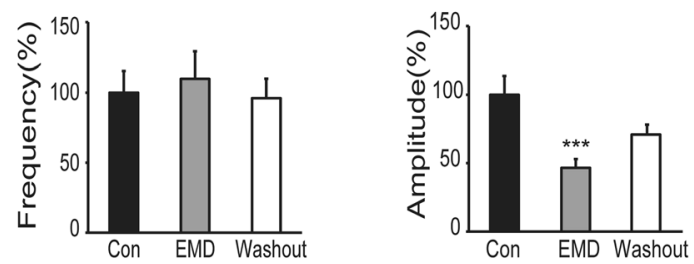

d
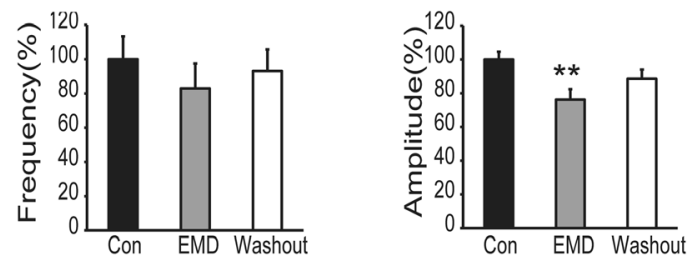

Fig. 3 Activation of 5-HT6 receptors increased the relative GABAergic neurotransmission on cortical pyramidal neurons. a Representative sEPSC traces. b Summarized data of EMD and its control $(n=6$ per group). $\mathbf{c}$ Representative sIPSC traces. d Summarized data of EMD and its control ( $n=8$ per group). Vertical bars represent the mean $\pm S E$. Asterisks show significant differences $(* * p<.01, * * * p<.001$ versus ACSF, one-way ANOVA/LSD post hoc test) 
a

Con

SB

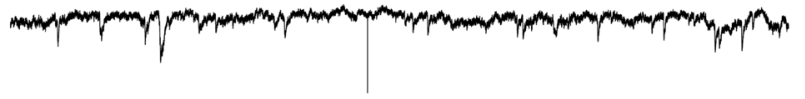

Washout

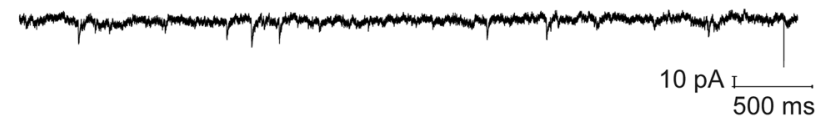

c

Con

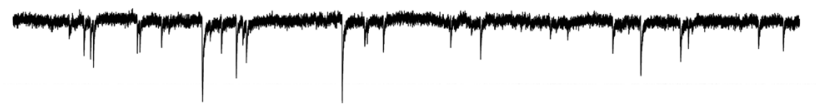

SB

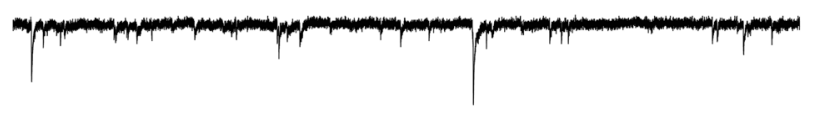

Washout

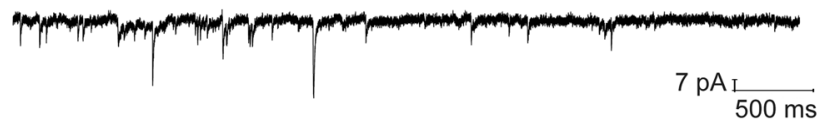

b
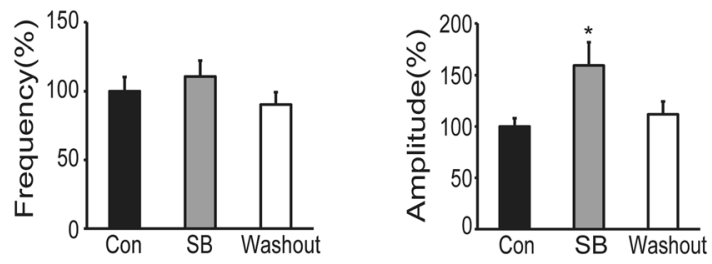

d
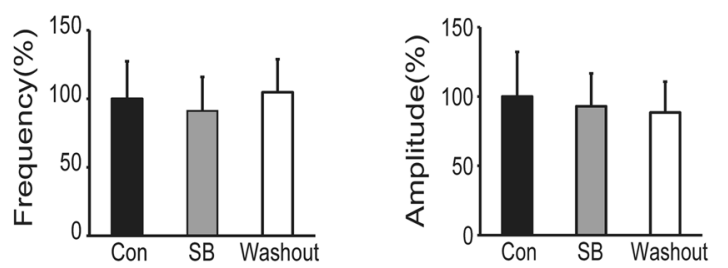

Fig. 4 Inactivation of 5-HT6 receptors increased the excitatory inputs on cortical pyramidal neurons. a Representative sEPSC traces. b Summarized data of SB and its control ( $n=6$ per group). c Representative sIPSC traces. d Summarized data of SB and its control

could reverse the anxiolytic effects of EMD, shown as the mice spent less time in the central region of the open-field test box, $F(3,39)=13.1, p=.0002$ (see Fig. 5a), less time in the open arms in elevated plus maze test, $F(3,39)=14.1, p<.001$ (see Fig. 5 c), and had a lower ratio in the social interaction test, $F(3$, 39 ) $=8.09, p<.001$ (see Fig. 5e). Conversely, the total distance they covered in the open field and their velocity in the elevate plus maze showed no significance, $F(3,39)=0.455, p=.715$ (see Fig. 5b); $F(3,39)=0.397, p=.756$ (see Fig. 5d).

When infused with BMI, there was no significant difference between the BMI group and mixed group, shown as time spent in the center, $F(3,39)=13.1, p=.065$ (see Fig. 5a), time spent in the open arms, $F(3,39)=14.1, p=.101$ (see Fig. 5c) and ratio in the social interaction test, $F(3,39)=8.09, p=.379$ (see Fig. 5e) had no significant effects. Furthermore, interactions between single doses of EMD and BMI (the open-field test, $p<.001$; the elevated plus maze test, $p=.0001$; the social interaction test, $p=.002$ ) were significant. These findings suggest that 5-HT6 receptors in the dmPFC may be important in the regulation of anxiety. Additionally, the relatively high GABAergic transmission by 5-HT6 receptor can be blocked by the $\mathrm{GABA}_{\mathrm{A}}$ receptor antagonist BMI.

\section{Rescue of anxiogenic behavior induced by SB via clonazepam treatment}

Given that the anxiogenic behavior induced by the infusion of $\mathrm{SB}$ arose from the relatively decreased GABAergic
( $n=8$ per group). Vertical bars represent the mean $\pm S E$. Asterisks show significant differences $\left({ }^{*} p<.05\right.$ versus ACSF, one-way ANOVA/LSD post hoc test)

transmission of the dmPFC pyramidal neuron, we reasoned that they could be rescued by increasing GABAergic transmission. To test this idea, we treated mice with clonazepam (CLZ) - a positive allosteric modulator of $\mathrm{GABA}_{\mathrm{A}}$ receptors. CLZ causes anxiolytic effects when the concentration was more than $0.0625 \mathrm{mg} / \mathrm{kg}$ (Han et al., 2012). CLZ can boost GABA signaling in the presence of presynaptically released GABA (Shah, Sjovold, \& Treit, 2004). A previous study reported that CLZ could increase the amplitude of SIPSC and decrease the frequency of sEPSC, which leads to increased strength of GABAergic transmission (Han et al., 2012).

Mice treated with CLZ and SB spent more time in the central area than did SB mice in the open-field test, $F(3,39)$ $=5.742, p=.048$ (see Fig. 6a); however, CLZ and SB infusion in the dmPFC did not affect the overall activity of the mice, as indicated by total path length in the open field test, $F(3,39)=$ $0.113, p=.951$ (see Fig. 6b). Additionally, CLZ and SB infusion increased time spent in the open arms, $F(3,39)=3.23, p$ $=.033$ (see Fig. $6 \mathrm{c}$ ) without affecting the velocity, $F(3,39)=$ $0.558, p=.646$ (see Fig. 6d). Furthermore, the ratio of the group with CLZ and SB infusion increased in the social interaction test, indicating the rescue of impaired social ability, $F(3,39)=11.423, p=.015$ (see Fig. 6e). Similarly, animals treated with CLZ $(0.5 \mathrm{mg} / \mathrm{kg}$, i.p.) explored for a longer time in the central area, $F(3,39)=5.74, p<.001$ (see Fig. 6a), spent more time in the open arms, $F(3,39)=3.23, p=.005$ (see Fig. $6 \mathrm{c})$, and had an increased ratio in the interaction test, $F(3,39)=$ 11.423, $p=.023$ (see Fig. 6e) compared to controls (Fig. 6a, 

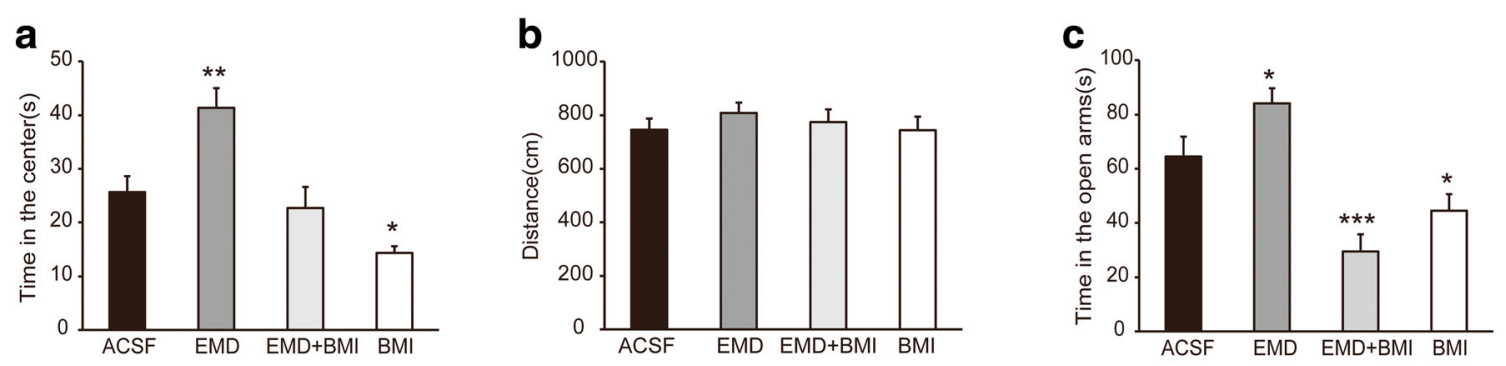

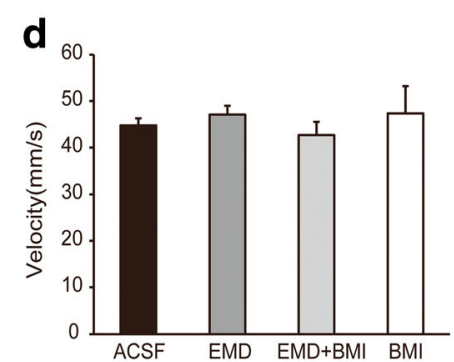

Fig. 5 Rescue of anxiolytic behavior induced by EMD via BMI infusion. Mice receiving BMI spent less time in the central region of the open field apparatus (a) and less time exploring the open arms of the elevated plus maze (c). BMI induced a smaller ratio in the social interaction test (e);

$6 \mathrm{c}$, and 6e). Furthermore, interactions between single doses of EMD and BMI (the open-field test, $p=.002$; the elevated plus maze test, $p=.003$; the social interaction test, $p<.001$ ) were

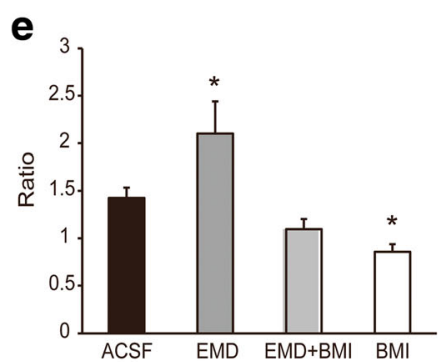

however, there was no difference in total traveling distance (b) and velocity (d). Data are presented as the mean $\pm S E . n=10$ mice per group. $* * p<.01, * * * p<.001$ versus ACSF, one-way ANOVA/LSD post hoc test

significant. These results indicate that a single dose of CLZ can reversibly rescue anxiogenic behavior through depression of 5-HT6 receptors.
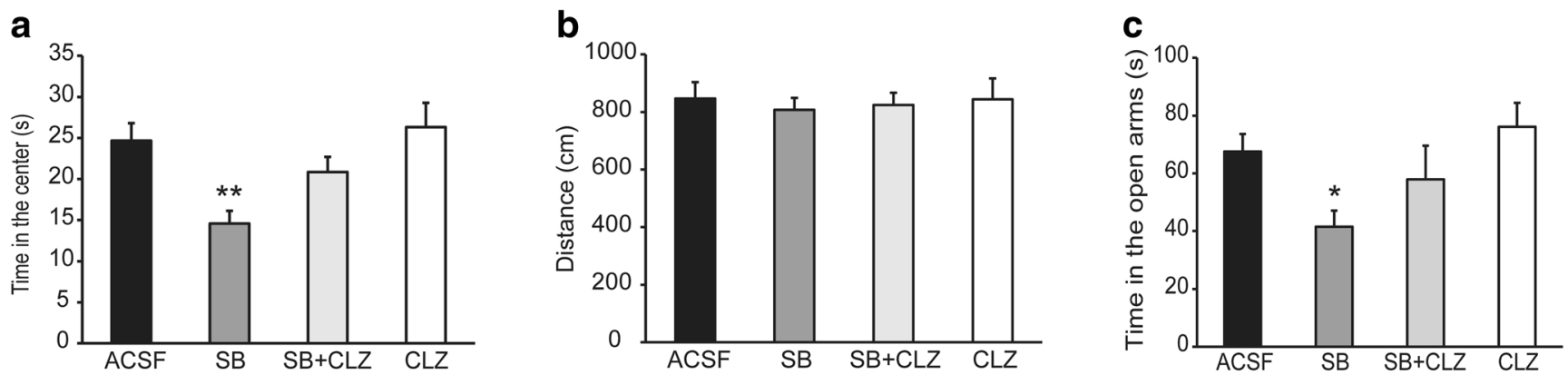

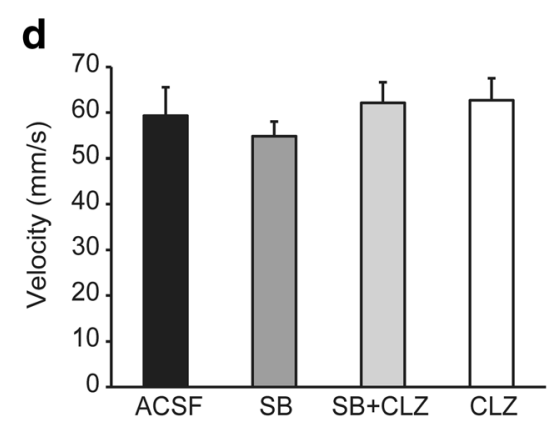

Fig. 6 Rescue of anxiogenic behavior induced by SB via clonazepam treatment. Mice co-treated with SB and CLZ spent more time in the central region of the open field apparatus (a) and more time exploring the open arms of the elevated plus maze (c). BMI induced a larger ratio in

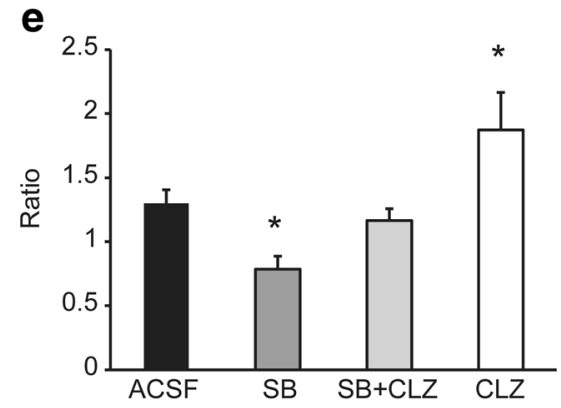

the social interaction test (e); however, no differences in total traveling distance (b) and velocity (d) were observed. Data are presented as the mean \pm SE. $n=10$ mice per group. $* p<.05, * * p<.01$ versus ACSF, oneway ANOVA/LSD post hoc test 


\section{Discussion}

The major findings of the present study were as follows. DmPFC 5-HT6 receptor activation increased the time spent in the central area in the open-field test, enhanced exploration of the open arms in the elevated plus maze test, and increased the ratio in the social interaction test; 5-HT6 receptor inactivation induced the opposite effects. In an acute brain slice, EMD both decreased spontaneous inhibitory postsynaptic currents (SIPSC) and spontaneous excitatory postsynaptic currents (sEPSC), while SB only increased sEPSC. These effects of EMD and SB could be reversed by the $\mathrm{GABA}_{\mathrm{A}}$ receptor antagonist bicuculline (BMI) and the positive allosteric modulator clonazepam. This may be the first study to demonstrate that dmPFC 5-HT6 receptors is critical for anxiety-like behavior, according to our knowledge.

The $\mathrm{mPFC}$ includes three regions: the prelimbic (PL) cortex, infralimbic (IL) cortex, and anterior cingulate (Cg1) cortex (Heidbreder \& Groenewegen, 2003). DmPFC is thought to regulate anxiety via glutamatergic projections to the central nucleus of the amygdala and bed nucleus of the stria terminalis (BNST) - key regions in the expression of anxiety responses (Lacroix, Spinelli, Heidbreder, \& Feldon, 2000; Maaswinkel, Gispen, \& Spruijt, 1996). We focused on the dmPFC due to its role in the regulation of anxiety (Covington et al., 2010; Jinks \& McGregor, 1997; Sabihi, Durosko, Dong, \& Leuner, 2014; Stack et al., 2010).

5-HT6 receptors are abundantly expressed in the frontal cortices, olfactory tubercle, dorsal hippocampus, striatum, and nucleus accumbens (Gerard et al., 1997; Roberts et al., 2002; Ward et al., 1995). 5-HT6 can regulate the release of other neurotransmitters in the brain, especially antagonists of 5-HT6 receptor which increase levels of forebrain glutamate, acetylcholine and norepinephrine (Dawson, Nguyen, \& Li, 2000, 2001; Riemer et al., 2003).

EMD is a potent 5-HT6-specific agonist. EMD increased practically all parameters of anxiety-related testing, without altering mice's locomotor activity; therefore, the anxiolyticlike responses of EMD cannot be explained by competing behaviors, such as the enhancement of general exploration. Our presented intra-dmPFC infusion results from EMD are in agreement with previous studies (Jastrzebska-Wiesek et al., 2014; Nikiforuk et al., 2011). Activation of 5-HT6 receptors through EMD infusion induced anxiolytic effects, and EMD was considered as a partial agonist (Jastrzebska-Wiesek et al., 2013). This posed an intriguing question of whether there are opposite effects after inactivation of 5-HT6 receptors, and so we checked the effects after stereotactic infusion of a selective 5-HT6 receptor antagonist, SB. After administration of SB, we observed anxiogenic effects and no effect on exploratory activity; these results seem to be directly related to the inhibition of 5-HT6 receptors.
The anxiolytic and anxiogenic behavior from EMD and SB may be caused by relative high or low GABAergic neurotransmission on dmPFC pyramidal neurons. Previous studies reported some electrophysiological properties of 5HT6 receptors. In medium spiny neurons, activation of 5HT6 receptors decreased the amplitude of spontaneous excitatory postsynaptic currents (Tassone et al., 2011). The 5-HT6 agonist WAY-181187 enhances the frequency of sIPSC in the hippocampus, and this increase was prevented by the 5-HT6 antagonist SB-399885 (West, Marcy, Marino, \& Schaffhauser, 2009). Our results showed an imbalance in excitatory and inhibitory transmission on mPFC pyramidal neurons. EMD reduced the amplitude of spontaneous EPSC and IPSC, but more so in EPSC, leading to a relative high GABAergic transmission level. SB increased the amplitude of spontaneous EPSC, maybe leading to a relative low GABAergic transmission. These results indicated that 5-HT6 receptors affect glutamatergic transmission or GABAergic transmission. We checked GABAergic transmission soon afterwards. Further studies looking at which glutamatergic receptors the 5-HT6 receptor affects and how it works remain to be investigated.

To further test our hypothesis, we treated mice with bicuculline (BMI) and clonazepam, in order to decrease and increase the GABAergic tone, respectively. High doses of benzodiazepine have been used to alleviate anxiety disorders (Low et al., 2000). Our results indicate that the effects of EMD and SB could be reversed by the $\mathrm{GABA}_{\mathrm{A}}$ receptor antagonist bicuculline (BMI) and positive allosteric modulator clonazepam, respectively. Here, GABAergic neurotransmission has been explored. However, we are unclear about the mechanisms of glutamate transmission. The detailed mechanisms remain to be investigated in future.

Interestingly, 5-HT6 receptor agonists and antagonists have been reported to produce similar effects in animal models of depression and anxiety. It is possible that 5HT6 receptor agonists and antagonists happen to produce similar behavioral effects through different neurochemical mechanisms or actions mediated in different brain regions. However, we could also observe opposite effects of the 5-HT6 antagonist SB-399885 (West et al., 2009). One must take into consideration that previous studies were administered intraperitoneally. Our intradmPFC results may provide another precisely localized explanation of the function of 5-HT6 receptor inhibition. More experiments should be addressed to explain the role of dmPFC 5-HT6 receptors in anxiety disorders in the future.

In conclusion, this study reveals the functional role of the dmPFC 5-HT6 receptor in anxiety, which maybe provide new insight for treatments of anxiety disorders. 
Acknowledgements This study was supported by China Postdoctoral Science Foundation Funded Project (2015M572336) and Guizhou Province Health and Family Planning Commission of Science and Technology Fund Project (gzwjkj2016-1-009). The authors declare no competing financial interests in relation to the work described.

\section{References}

Brailov, I., Bancila, M., Brisorgueil, M. J., Miquel, M. C., Hamon, M., \& Verge, D. (2000). Localization of 5-HT(6) receptors at the plasma membrane of neuronal cilia in the rat brain. Brain Research, 872(1/2), 271-275.

Carr, G. V., Schechter, L. E., \& Lucki, I. (2011). Antidepressant and anxiolytic effects of selective 5-HT6 receptor agonists in rats. Psychopharmacology, 213(2/3), 499-507. https://doi.org/10.1007/ s00213-010-1798-7

Courtin, J., Chaudun, F., Rozeske, R. R., Karalis, N., Gonzalez-Campo, C., Wurtz, H., ... Herry, C. (2014). Prefrontal parvalbumin interneurons shape neuronal activity to drive fear expression. Nature, 505(7481), 92-96. https://doi.org/10.1038/nature12755

Covington, H. E., III, Lobo, M. K., Maze, I., Vialou, V., Hyman, J. M., Zaman, S., ... Nestler, E. J. (2010). Antidepressant effect of optogenetic stimulation of the medial prefrontal cortex. Journal of Neuroscience, 30(48), 16082-16090. https://doi.org/10.1523/ jneurosci.1731-10.2010

Dawson, L. A., Nguyen, H. Q., \& Li, P. (2000). In vivo effects of the 5HT(6) antagonist SB-271046 on striatal and frontal cortex extracellular concentrations of noradrenaline, dopamine, 5-HT, glutamate and aspartate. British Journal of Pharmacology, 130(1), 23-26. https://doi.org/10.1038/sj.bjp.0703288

Dawson, L. A., Nguyen, H. Q., \& Li, P. (2001). The 5-HT(6) receptor antagonist SB-271046 selectively enhances excitatory neurotransmission in the rat frontal cortex and hippocampus. Neuropsychopharmacology, 25(5), 662-668. https://doi.org/10. 1016/s0893-133x(01)00265-2

Geng, F., Zhang, J., Wu, J. L., Zou, W. J., Liang, Z. P., Bi, L. L., ... Gao, T. M. (2016). Neuregulin 1-ErbB4 signaling in the bed nucleus of the stria terminalis regulates anxiety-like behavior. Neuroscience, 329, 182-192. https://doi.org/10.1016/j.neuroscience.2016.05.018

Gerard, C., Martres, M. P., Lefevre, K., Miquel, M. C., Verge, D., Lanfumey, L., ... el Mestikawy, S. (1997). Immuno-localization of serotonin 5-HT6 receptor-like material in the rat central nervous system Brain Research, 746(1/2), 207-219.

Haber, S. N., \& Behrens, T. E. (2014). The neural network underlying incentive-based learning: Implications for interpreting circuit disruptions in psychiatric disorders. Neuron, 83(5), 1019-1039. https:// doi.org/10.1016/j.neuron.2014.08.031

Hamon, M., Doucet, E., Lefevre, K., Miquel, M. C., Lanfumey, L., Insausti, R., ... Verge, D. (1999). Antibodies and antisense oligonucleotide for probing the distribution and putative functions of central 5-HT6 receptors. Neuropsychopharmacology, 21(2, Suppl,), 68S76S. doi:https://doi.org/10.1016/s0893-133x(99)00044-5

Han, S., Tai, C., Westenbroek, R. E., Yu, F. H., Cheah, C. S., Potter, G. B., ... Catterall, W. A. (2012). Autistic-like behaviour in Scn1a+/- mice and rescue by enhanced GABA-mediated neurotransmission. Nature, 489(7416), 385-390. https://doi.org/10.1038/nature11356

Heal, D. J., Smith, S. L., Fisas, A., Codony, X., \& Buschmann, H. (2008). Selective 5-HT6 receptor ligands: Progress in the development of a novel pharmacological approach to the treatment of obesity and related metabolic disorders. Pharmacology and Therapeutics, 117(2), 207-231. https://doi.org/10.1016/j. pharmthera.2007.08.006
Heidbreder, C. A., \& Groenewegen, H. J. (2003). The medial prefrontal cortex in the rat: Evidence for a dorso-ventral distinction based upon functional and anatomical characteristics Neuroscience \& Biobehavioral Review, 27(6), 555-579

Jastrzebska-Wiesek, M., Siwek, A., Kazek, G., Nawiesniak, B., Partyka, A., Marcinkowska, M., ... Wesolowska, A. (2013). Partial agonist efficacy of EMD386088, a 5-HT6 receptor ligand, in functional in vitro assays. Pharmacology Reports, 65(4), 998-1005

Jastrzebska-Wiesek, M., Siwek, A., Partyka, A., Kubacka, M., Mogilski, S., Wasik, A., ... Wesolowska, A. (2014). Pharmacological evaluation of the anxiolytic-like effects of EMD 386088, a partial 5-HT6 receptor agonist, in the rat elevated plus-maze and Vogel conflict tests. Neuropharmacology, 85, 253-262. https://doi.org/10.1016/j. neuropharm.2014.05.036

Jinks, A. L., \& McGregor, I. S. (1997). Modulation of anxiety-related behaviours following lesions of the prelimbic or infralimbic cortex in the rat. Brain Research, 772(1/2), 181-190.

Kober, H., Barrett, L. F., Joseph, J., Bliss-Moreau, E., Lindquist, K., \& Wager, T. D. (2008). Functional grouping and cortical-subcortical interactions in emotion: A meta-analysis of neuroimaging studies. NeuroImage, 42(2), 998-1031. https://doi.org/10.1016/j. neuroimage.2008.03.059

Lacroix, L., Spinelli, S., Heidbreder, C. A., \& Feldon, J. (2000). Differential role of the medial and lateral prefrontal cortices in fear and anxiety. Behavioral Neuroscience, 114(6), 1119-1130.

Low, K., Crestani, F., Keist, R., Benke, D., Brunig, I., Benson, J. A., ... Rudolph, U. (2000). Molecular and neuronal substrate for the selective attenuation of anxiety. Science, 290(5489), 131-134.

Maaswinkel, H., Gispen, W. H., \& Spruijt, B. M. (1996). Effects of an electrolytic lesion of the prelimbic area on anxiety-related and $\operatorname{cog}$ nitive tasks in the rat. Behavioral Brain Research, 79(1/2), 51-59.

Maier, S., Szalkowski, A., Kamphausen, S., Perlov, E., Feige, B., Blechert, J., ... Tuscher, O. (2012). Clarifying the role of the rostral $\mathrm{dmPFC} / \mathrm{dACC}$ in fear/anxiety: Learning, appraisal or expression? PLOS ONE, 7(11), e50120. https://doi.org/10.1371/journal.pone. 0050120

Mitchell, E. S., \& Neumaier, J. F. (2005). 5-HT6 receptors: A novel target for cognitive enhancement. Pharmacolgy and Therapeutics, 108(3), 320-333. https://doi.org/10.1016/j.pharmthera.2005.05.001

Nikiforuk, A., Kos, T., \& Wesolowska, A. (2011). The 5-HT6 receptor agonist EMD 386088 produces antidepressant and anxiolytic effects in rats after intrahippocampal administration. Psychopharmacology, 217(3), 411-418. https://doi.org/10.1007/s00213-011-2297-1

Pogacic Kramp, V. (2012). List of drugs in development for neurodegenerative diseases: Update October 2011. Neurodegenerative Diseases, 9(4), 210-283. https://doi.org/10.1159/000335520

Riemer, C., Borroni, E., Levet-Trafit, B., Martin, J. R., Poli, S., Porter, R. H., \& Bos, M. (2003). Influence of the 5-HT6 receptor on acetylcholine release in the cortex: Pharmacological characterization of 4 (2-bromo-6-pyrrolidin-1-ylpyridine-4-sulfonyl)phenylamine, a potent and selective 5-HT6 receptor antagonist Journal of Medicinal Chemistry, 46(7), 1273-1276. https://doi.org/10.1021/jm021085c

Roberts, J. C., Reavill, C., East, S. Z., Harrison, P. J., Patel, S., Routledge, C., \& Leslie, R. A. (2002). The distribution of 5-HT(6) receptors in rat brain: An autoradiographic binding study using the radiolabelled 5-HT(6) receptor antagonist [(125)I]SB-258585. Brain Research, 934(1), 49-57.

Rogers, D. C., \& Hagan, J. J. (2001). 5-HT6 receptor antagonists enhance retention of a water maze task in the rat. Psychopharmacology, 158(2), 114-119. https://doi.org/10.1007/s002130100840

Ruat, M., Traiffort, E., Arrang, J. M., Tardivel-Lacombe, J., Diaz, J., Leurs, R., \& Schwartz, J. C. (1993). A novel rat serotonin (5-HT6) receptor: Molecular cloning, localization and stimulation of cAMP accumulation. Biochemical and Biophysical Research Communications, 193(1), 268-276. 
Rushworth, M. F., \& Behrens, T. E. (2008). Choice, uncertainty and value in prefrontal and cingulate cortex. Nature Neuroscience, 11(4), 389397. https://doi.org/10.1038/nn2066

Rushworth, M. F., Noonan, M. P., Boorman, E. D., Walton, M. E., \& Behrens, T. E. (2011). Frontal cortex and reward-guided learning and decision-making. Neuron, 70(6), 1054-1069. https://doi.org/ 10.1016/j.neuron.2011.05.014

Sabihi, S., Durosko, N. E., Dong, S. M., \& Leuner, B. (2014). Oxytocin in the prelimbic medial prefrontal cortex reduces anxiety-like behavior in female and male rats. Psychoneuroendocrinology, 45, 31-42. https://doi.org/10.1016/j.psyneuen.2014.03.009

Schechter, L. E., Lin, Q., Smith, D. L., Zhang, G., Shan, Q., Platt, B., ... Beyer, C. E. (2008). Neuropharmacological profile of novel and selective 5-HT6 receptor agonists: WAY-181187 and WAY208466. Neuropsychopharmacology, 33(6), 1323-1335. https:// doi.org/10.1038/sj.npp.1301503

Shah, A. A., Sjovold, T., \& Treit, D. (2004). Inactivation of the medial prefrontal cortex with the GABAA receptor agonist muscimol increases open-arm activity in the elevated plus-maze and attenuates shock-probe burying in rats. Brain Research, 1028(1), 112-115. https://doi.org/10.1016/j.brainres.2004.08.061

Solati, J., Hajikhani, R., \& Golub, Y. (2013). Activation of GABAA receptors in the medial prefrontal cortex produces an anxiolyticlike response. Acta Neuropsychiatr, 25(4), 221-226. https://doi. org/10.1111/acn.12016
Stack, A., Carrier, N., Dietz, D., Hollis, F., Sorenson, J., \& Kabbaj, M. (2010). Sex differences in social interaction in rats: Role of the immediate-early gene zif268. Neuropsychopharmacology, 35(2), 570-580. https://doi.org/10.1038/npp.2009.163

Tassone, A., Madeo, G., Schirinzi, T., Vita, D., Puglisi, F., Ponterio, G., ... Bonsi, P. (2011). Activation of 5-HT6 receptors inhibits corticostriatal glutamatergic transmission. Neuropharmacology, 61(4), 632-637. https://doi.org/10.1016/j.neuropharm.2011.05.004

Vogt, B. A., Finch, D. M., \& Olson, C. R. (1992). Functional heterogeneity in cingulate cortex: The anterior executive and posterior evaluative regions. Cerebral Cortex, 2(6), 435-443.

Ward, R. P., Hamblin, M. W., Lachowicz, J. E., Hoffman, B. J., Sibley, D. R., \& Dorsa, D. M. (1995). Localization of serotonin subtype 6 receptor messenger RNA in the rat brain by in situ hybridization histochemistry. Neuroscience, 64(4), 1105-1111.

Wesolowska, A., \& Nikiforuk, A. (2007). Effects of the brain-penetrant and selective 5-HT6 receptor antagonist SB-399885 in animal models of anxiety and depression. Neuropharmacology, 52(5), 1274-1283. https://doi.org/10.1016/j.neuropharm.2007.01.007

West, P. J., Marcy, V. R., Marino, M. J., \& Schaffhauser, H. (2009). Activation of the 5-HT(6) receptor attenuates long-term potentiation and facilitates GABAergic neurotransmission in rat hippocampus. Neuroscience, 164(2), 692-701. https://doi.org/10.1016/j. neuroscience.2009.07.061 